\title{
Cochlear Homocysteine Metabolism at the Crossroad of Nutrition and Sensorineural Hearing Loss
}

\author{
Teresa Partearroyo ${ }^{1}$, Néstor Vallecillo ${ }^{2,3}$, María A. Pajares ${ }^{2,4}$, Gregorio Varela-Moreiras ${ }^{1}$ \\ and Isabel Varela-Nieto ${ }^{2,3,4 *}$ \\ 'Departamento de Ciencias Farmacéuticas y de la Salud, Facultad de Farmacia, Universidad CEU San Pablo, Madrid, Spain, \\ ${ }^{2}$ Departamento de Fisiopatología y del Sistema Nervios, Instituto de Investigaciones Biomédicas Alberto Sols, Consejo \\ Superior de Investigaciones Científicas (CSIC-UAM), Madrid, Spain, ${ }^{3}$ Centro de Investigación Biomédica en Red de \\ Enfermedades Raras (CIBERER), Instituto de Salud Carlos III, Madrid, Spain, ${ }^{4}$ Investigación en Otoneurocirugía, Instituto de \\ Investigación Sanitaria La Paz (IdiPAZ), Madrid, Spain
}

Hearing loss $(\mathrm{HL})$ is one of the most common causes of disability, affecting 360 million people according to the World Health Organization (WHO). HL is most frequently of sensorineural origin, being caused by the irreversible loss of hair cells and/or spiral ganglion neurons. The etiology of sensorineural $\mathrm{HL}(\mathrm{SNHL})$ is multifactorial, with genetic and environmental factors such as noise, ototoxic substances and aging playing a role. The nutritional status is central in aging disability, but the interplay between nutrition and SNHL has only recently gained attention. Dietary supplementation could therefore constitute the first step for the prevention and potential repair of hearing damage before it reaches irreversibility. In this context, different epidemiological studies have shown correlations among the nutritional condition, increased total plasma homocysteine (tHcy) and SNHL. Several human genetic rare diseases are also associated with homocysteine (Hcy) metabolism and SNHL confirming this potential link. Accordingly, rodent experimental models have provided the molecular basis to understand the observed effects. Thus, increased tHcy levels and vitamin deficiencies, such as folic acid (FA), have been linked with $\mathrm{SNHL}$, whereas long-term dietary supplementation with omega-3 fatty acids improved Hcy metabolism, cell survival and hearing acuity. Furthermore, pharmacological supplementations with the anti-oxidant fumaric acid that targets Hcy metabolism also improved SNHL. Overall these results strongly suggest that cochlear Hcy metabolism is a key player in the onset and progression of SNHL, opening the way for the design of prospective nutritional therapies.

*Correspondence: Isabel Varela-Nieto ivarela@iib.uam.es

Received: 26 January 2017 Accepted: 30 March 2017

Published: 25 April 2017

Citation:

Partearroyo T, Vallecillo $N$, Pajares MA, Varela-Moreiras $G$ and Varela-Nieto I (2017) Cochlear Homocysteine Metabolism at the Crossroad of Nutrition and Sensorineural Hearing Loss.

Front. Mol. Neurosci. 10:107. doi: 10.3389/fnmol.2017.00107

\section{Keywords: folic acid, omega-3, nutritional imbalance, one-carbon metabolism, oxidative stress, rare diseases}

\section{HEARING LOSS}

According to the World Health Organization (2015), moderate-to-profound Hearing loss (HL) affects 360 million people worldwide. Its incidence varies in each population segment, affecting already $\sim 10 \%$ of children and increasing to $30 \%$ of the population over 65 years (Roth et al., 2011; Li-Korotky, 2012). In addition, HL has been ranked as the fifth leading cause of years lived with disability in the Global Burden of Disease Study 2013 Collaborators (2015). Thus, this impairment certainly limits the quality of life, and significantly increases the risk of dependance. Therefore, the identification of factors involved in HL is key to understand the physiopathology, to improve diagnosis and to develop appropriate therapies and preventive behaviors. 
The ear consists of three parts of which the cochlea, in the inner ear, is responsible for the mechanotransduction of the sound stimulus. At the cochlea, in the scala media, more than a dozen interconnected cell types are fundamental for hearing. The auditory sensory epithelium, the organ of Corti, contains two types of hair cells, outer and inner, innervated by the spiral ganglion neurons that connect it with the brain (Magariños et al., 2012, 2014). The irreversible loss of hair cells and/or neurons, or their malfunctions are the typical causes of sensorineural HL (SNHL).

SNHL has a multifactorial origin that combines genetic with environmental factors (Dror and Avraham, 2009; Roth et al., 2011). Genetic factors comprise mutations in genes or regulatory elements involved in the development, structure or function of the cochlea. For example, a fundamental role in hearing is played by proteins involved in cell-cell junctions (tight junctions, adherent junctions and gap junctions), and among them the connexins. Mutations in five of the 21 genes of the human connexin family have been linked to the onset of deafness (Cx26,Cx30,Cx31,Cx32 and Cx43; Dror and Avraham, 2009). Moreover, knockdown of one of these genes, precisely the $\mathrm{Cx} 30$, correlates with alterations in cochlear homocysteine (Hcy) metabolism (Cohen-Salmon et al., 2007). Conversely, hyperhomocysteinemia (HHcy) increases the levels of $\mathrm{Cx} 43$ in a variety of cellular contexts (Li et al., 2002; Boot et al., 2006). Interestingly, Cbs heterozygous mice show HHcy and impaired matrix remodeling in the cochlea (Kundu et al., 2009). Cell-cell contacts are essential for hearing and, therefore, Hcy accumulation could have deleterious effects on the hearing receptor machinery. Further studies are needed to fully understand the role of $\mathrm{Hcy}$ and its metabolites in the cochlea. On the other hand, environmental factors include exposure to high levels of noise, ototoxic drugs or nutritional deficiencies (Gok et al., 2004; Tabuchi et al., 2011). Moreover, certain genetic factors predispose to suffer damage due to noise or ototoxic drugs, as well as, to premature aging of the hearing structures (Zhao et al., 2004; Rydzanicz et al., 2010); their identification is one of the current challenges in this field of research. Approximately $50 \%$ of the cases of hereditary SNHL are accompanied by other clinical symptoms, and statistically 1 in every 1000 newborns has profound deafness and one more will suffer HL before adulthood. Altogether, these data suggest that manipulation of micronutrients could be a tool to understand the genes and physiopathological mechanisms involved in hearing and SNHL.

\section{HYPERHOMOCYSTEINEMIA AND NEUROSENSORIAL HEARING LOSS}

HHcy is an acquired metabolic problem that was first described by McCully (1969), and whose interest increased greatly due to the detection of high plasmatic levels of Hcy (tHcy) in cardiovascular diseases (Ueland and Refsum, 1989). Similarly, HHcy also shows a strong correlation with the development of neurological disorders, chronic kidney disease, osteoporosis, gastrointestinal disorders, cancer and the presence of certain congenital defects (Givvimani et al., 2012; Schalinske and Smazal, 2012; Iacobazzi et al., 2014; Lehotsky et al., 2015; Perna and Ingrosso, 2016). In fact, high levels of tHcy are quite common, and are detected in $10 \%-20 \%$ of the population as a result of genetic (Brosnan et al., 2004) and other factors (Refsum et al., 1989; Noga et al., 2003), but their incidence depends widely on geography, age, sex and ethnicity (Yang et al., 2014).

Regarding hearing impairment and HHcy, several epidemiological studies have shown an association between certain nutritional deficiencies and development of SNHL. Reduced folic acid (FA) concentrations have been found in age-related HL (ARHL) and sudden SNHL, this decrease correlating with either reduced vitamin $\mathrm{B}_{12}$ (Houston et al., 1999; Lasisi et al., 2010; Karli et al., 2013) or increased tHcy levels (Cadoni et al., 2004). Although Hcy concentrations reflect alterations in its metabolism (Figure 1), which include the methionine and folate cycles, as well as the transsulfuration pathway, no consistent association between mutations in genes of these pathways and SNHL has been reported (Durga et al., 2006; Uchida et al., 2011). Analysis of the putative relationship between the methylenetetrahydrofolate reductase (MTHFR) C677T mutation and ARHL rendered contradictory results (Durga et al., 2006), since this allele has been correlated with hearing impairment and HHcy, and also with a reduced risk of ARHL independent from folate and Hcy levels (Uchida et al., 2011; Fusconi et al., 2012).

Epidemiological research has also provided evidence associating atherosclerosis in the inner ear and poor hearing, and connecting risk factors of vascular disease and ARHL (Rosen and Olin, 1965; Johnsson and Hawkins, 1972; Makishima, 1978). Among them, Hcy is also an agonist of N-methyl-D-aspartate receptors, which are overexcited in SNHL (Puel et al., 1991; Lipton et al., 1997). Thus, the mechanisms linking SNHL and Hcy metabolism seem to include a large variety of pathways, their connections remaining underexplored.

Hcy emerges as a node of key pathways of the intermediary metabolism that has been mainly explored in the liver (Figure 1), whereas knowledge of these routes and their regulation has been scarcely analyzed in peripheral or neurosensory organs (Pajares and Pérez-Sala, 2006; Obeid, 2013). In fact, the cochlea is one of the few sensory organs in which a whole expression and protein profile of the methionine cycle and transsultfuration pathway has been reported (Martínez-Vega et al., 2015a). Based on the hepatic knowledge, attempts to decrease the systemic tHcy levels by the design of several supplementation studies have been carried out. These randomized trials demonstrated the lowering of tHcy levels by dietary folate supplementation (Jacques et al., 1999), and provided the basis for an intervention trial carried out by Durga et al. (2007) to assess effects of folate supplementation on ARHL. The results obtained showed a slower progression of ARHL in individuals receiving the supplement.

SNHL has been associated with rare diseases involving alterations in Hcy levels. This is the case of combined methylmalonic acidemia and homocystinuria cblC type 


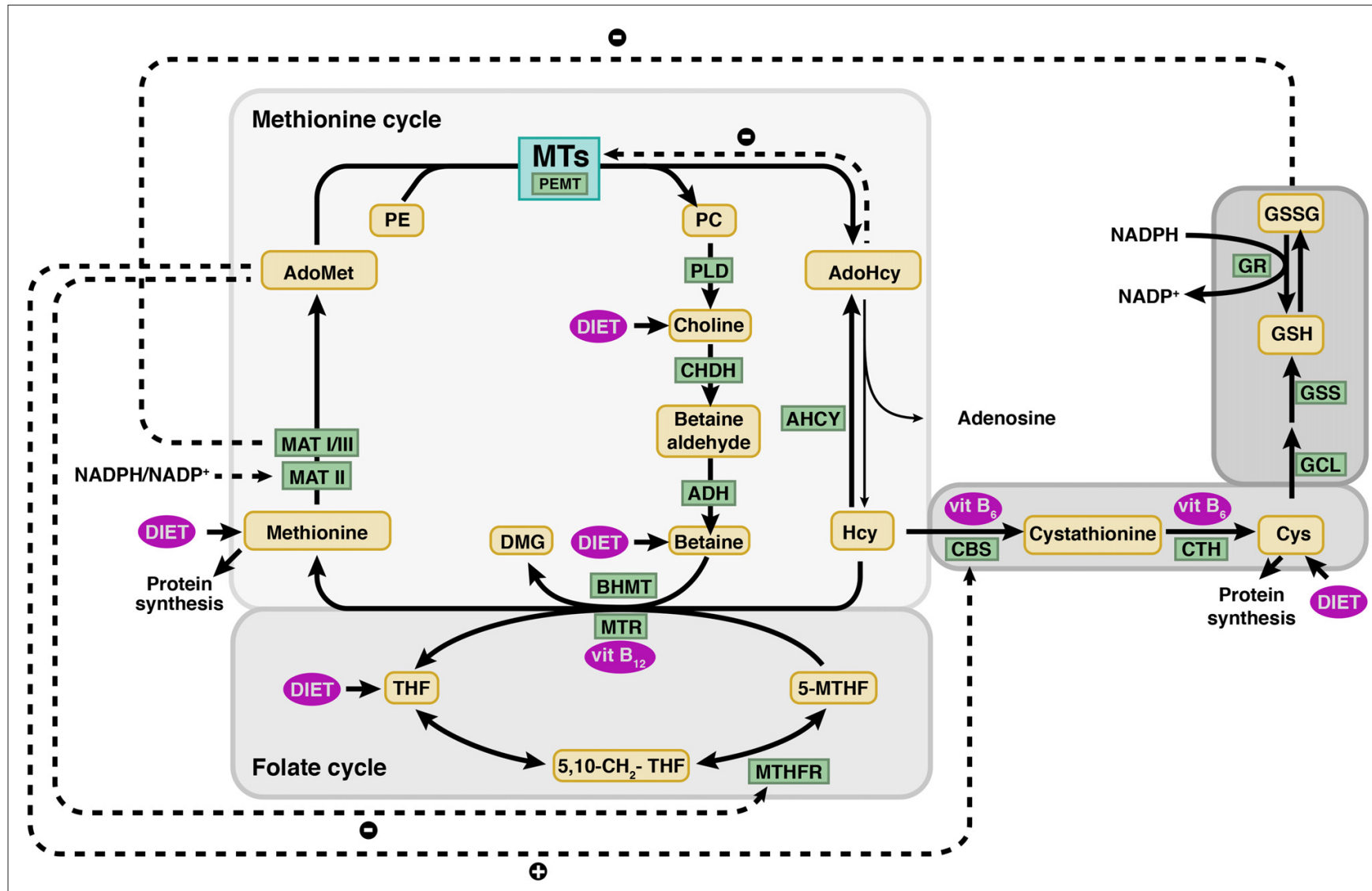

FIGURE 1 | Methionine and folate metabolism and connecting pathways. Schematic view of the main metabolic reactions involved in homocysteine (Hcy) metabolism, highlighting the major regulatory mechanisms (dotted lines). Hcy remethylation is part of the methionine cycle and is catalyzed by the vitamin $\mathrm{B}_{12}$ dependent methionine synthase (MTR) or betaine homocysteine methyltransferase (BHMT), enzymes that generate methionine using $5^{\prime}$-methyltetrahydrofolate (5-MTHF) and betaine as methyl donors, respectively. Methionine adenosyltransferases (MATs: MAT I, II and III) use methionine to synthesize S-adenosylmethionine (AdoMet). Donation of the AdoMet methyl group renders S-adenosylhomocysteine (AdoHcy) that is hydrolyzed by S-adenosylhomocysteine hydrolase (AHCY) to produce Hcy and adenosine in a reversible reaction. Hcy catabolism takes place initially by serine conjugation, a reaction catalyzed by cystathionine $\beta$-synthase (CBS) and that lead to cystathionine synthesis. This metabolite is then utilized by cystathionine $\gamma$-lyase (CTH) to produce cysteine (Cys). Both reactions depend on pyridoxal phosphate (vitamin $B_{6}$ ). The correct function of these pathways depends on a continuous supply of nutrients, methionine, vitamins $B_{12}$ and $B_{6}$ and folate. The latter is used in the folate cycle for the synthesis of 5-MTHF catalyzed by methylenetetrahydrofolate reductase (MTHFR). A reduced ingestion of the aforementioned nutrients leads to a decrease in the flux through these pathways, in which many cellular key compounds are generated (phospholipids, neurotransmitters, etc.). Enzymes and metabolites appear in square and rounded boxes, respectively. $\mathrm{ADH}$, aldehyde dehydrogenase; $\mathrm{CHDH}$, choline oxidase; 5,10- $\mathrm{CH}_{2}$-THF, 5,10-methylenetetrahydrofolate; DMG, dimethylglycine; GCL, glutamate-cysteine ligase; GR, glutathione reductase; GSH, reduced glutathione; GSS, glutathione synthase; GSSG, oxidized glutathione; MTs, methyltransferases; NADP ${ }^{+}$, nicotinamide adenine dinucleotide phosphate; NADPH, reduced form of $\mathrm{NADP}^{+}$; PC, phosphatidylcholine; PE, phosphatidylethanolamine; PEMT, phosphatidylethanolanime N-methyltransferase; PLD, phospholipase D; THF, tetrahydrofolate; vit $\mathrm{B}_{6}$, vitamin $\mathrm{B}_{6}$; vit. $\mathrm{B}_{12}$, vitamin $\mathrm{B}_{12}$.

(OMIM 277400), caused by mutations in the MMACHC gene. These mutations result in a decreased production of cofactors for methylmalonyl-CoA mutase (adenosylcobalamin) and methionine synthase (MTR; methylcobalamin) and, in turn, in elevated Hcy levels in the cerebrospinal fluid that correlate with unilateral SNHL (Harding et al., 2003; Tsai et al., 2007; Carrillo-Carrasco et al., 2012). Another rare disease reported to result with SNHL and homocystinuria is spastic quadriplegia, retinitis pigmentosa and mental retardation (OMIM 270950; Gordon et al., 1976), but this consanguinity disorder has been described in a very limited number of cases, and hence, the common molecular bases remain unknown. Among patients with osteogenesis imperfecta type I (OMIM 166200), which present with mutations in several genes including COL1A1 and COL1A2, that codify for the $\alpha 1$ and $\alpha 2$ chains of type I collagen, a correlation with SNHL has been also detected from the age of 20-years onward (Hartikka et al., 2004). Hcy is known to decrease the H3K9me2 content on the COL1A1 gene promoter leading to its upregulation. This effect is dependent on Hcy inhibition of G9a histone methyltransferase expression, which in turn results in lower G9a binding to the neuronrestrictive silencer element (NRSE) of this promoter (Lei et al., 2015). Additionally, Hcy has been also shown to increase oxidative mechanisms and activation of mitochondrial matrix metalloproteinase causing bone matrix degradation and 
alterations in its biomechanical properties (Behera et al., 2016), facts that could affect mechanotransduction of the sound in the middle ear.

Further studies in animal models confirmed the association between SNHL and HHcy. For example, Cbs ${ }^{+/-}$ heterozygous mice show moderate SNHL, high levels of Hcy in the stria vascularis and the spiral ligament, oxidative stress and reduction of vessel density, effects that were prevented by the administration of $\mathrm{FA}$ in the drinking water (Kundu et al., 2012). In $C \times 30^{-/-}$null mice hearing impairment was also associated with alterations in the stria vascularis, precisely down- and up-regulation of Betaine homocysteine methyltransferase (BHMT) and Ahcy expression, respectively, and increased Hcy immunostaining (Cohen-Salmon et al., 2007). All these data, epidemiological and derived from human and mouse genetic studies, reinforced the idea of the importance of Hcy metabolism in deafness.

\section{NUTRITIONAL INTERVENTIONS AND HEARING LOSS}

To date, supplementation and nutrient deficiency studies have been conducted using laboratory animals, in which auditory thresholds, internal ear microvasculature, or inflammation have been evaluated (Schuknecht et al., 1974; Makishima, 1978); but only a few have analyzed Hcy metabolism following the lead of the epidemiological data (Houston et al., 1999; Cadoni et al., 2004; Lasisi et al., 2010). Two studies in mouse models have shown the relationship between Hcy metabolism and SNHL and the impact of folate deficiency (FD; MartínezVega et al., 2015a, 2016). C57BL/6J mice, a mouse strain prone to SNHL, showed increased hearing thresholds after 2 months on a FD diet (Martínez-Vega et al., 2015a). This reduced intake of folate caused decreased serum concentrations of this micronutrient and increased tHcy. These systemic alterations correlated with SNHL, which also coincided with changes in expression and protein levels of cochlear Hcy metabolism aimed at decreasing the production of this amino acid, avoid or moderate its remethylation and catabolism, and induce its elimination into the plasma (Martínez-Vega et al., 2015a).

However, these cellular efforts to balance intracellular levels of $\mathrm{Hcy}$ are not sufficient as increased protein $\mathrm{N}$-homocysteinylation was detected in cochlear whole extracts; such post-translational modification may contribute to the inactivation and aggregation of proteins (Sharma et al., 2015). The reduced Hcy utilization by the transsulfuration pathway has an additional negative effect by decreasing $\mathrm{H}_{2} \mathrm{~S}$ production via CBS and cystathionine $\gamma$ lyase $(\mathrm{CTH})$. The protective role of this gasotransmitter as cochlear vasodilator has been reported in noise-induced HL (Li et al., 2011), and hence, the putative contribution of its reduced levels to the changes described in the microvasculature (Prazma et al., 1990; Gratton and Schulte, 1995). These changes are accompanied by a moderate increase in cochlear oxidative stress, as it is usually the case in the aging process.

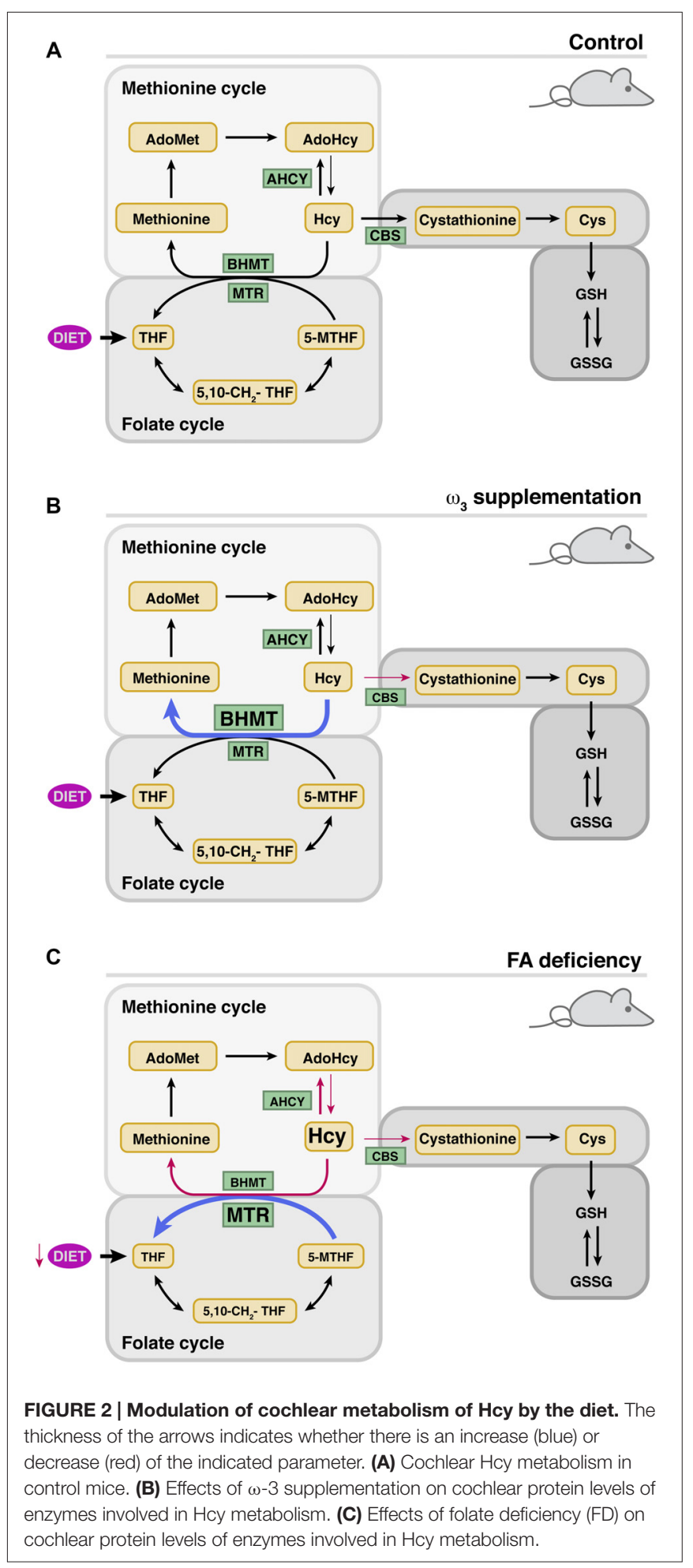

Altogether, these findings demonstrate that the relationship between HHcy induced by FD and premature SNHL involves cellular degeneration, impairment of cochlear Hcy metabolism and associated oxidative stress (Martínez-Vega et al., 2015a). Moreover, these results were later confirmed in a long-term study on the effects of FD using a mouse strain with delayed 
SNHL onset, in which, again, the deficiency caused premature SNHL (Martínez-Vega et al., 2016). The latter work also evidenced that the consequences of nutritional imbalances are strongly dependent on the mouse genetic background. Parallel evidences supporting the importance of nutrition on HL come from studies using injection of rodents with a variety of antioxidants, which showed different degrees of otic protection (Fetoni et al., 2010, 2014, 2015). On the other hand, it should be mentioned that an overdose of anti-oxidants may have undesired secondary consequences (Mancuso, 2015).

Human studies have also provided evidences of the putative prevention of SNHL by dietary supplementation with different levels of n-3 polyunsaturated ( $\omega 3$ ) fatty acids (Rosen et al., 1970; Dullemeijer et al., 2010; Gopinath et al., 2010; Stefanutti et al., 2013). Fish oil supplementation resulted in an inverse correlation between the ingested levels of long-chain $\omega 3$ fatty acids and SNHL (Dullemeijer et al., 2010; Gopinath et al., 2010). Moreover, Curhan et al. (2014) demonstrated that regular fish consumption ( 2 or more servings of fish per week) and higher intake of $\omega 3$ are associated with a lower risk of HL in women, but had opposite effects on tHcy (Piolot et al., 2003; Huang et al., 2011). Dissimilar results on tHcy and/or hearing thresholds were also obtained from animal studies of $\omega 3$-suplementation carried out for limited time periods in adults or during pregnancy and lactation (Church et al., 2007, 2009, 2010; Kulkarni et al., 2011; Huang et al., 2013). A recent study focused on analyzing the effects of long-term administration of a polyunsaturated fatty acid (PUFA)-rich diet on cochlear Hcy metabolism showed limited SNHL prevention with no changes in serum folate levels or tHcy (Martínez-Vega et al., 2015b; Figure 2). Nevertheless, this diet prevented changes in cochlear expression of age-induced Hcy metabolism genes (Bhmt and Cbs) and, importantly, the altered expression of proinflammatory cytokines was precluded, and the cochlear cytoarchitecture maintained. However, there was an increase in BHMT protein levels, an enzyme involved not only in the conservation of methionine levels, but also in recycling the choline derived from phospholipid metabolism (Martínez-Vega et al., 2015b).

Altogether, these data could suggest that the diet supplemented with $\omega 3$ induces a moderate increase in intracellular Hcy levels, although the lack of significant alterations in cochlear homocysteinylated protein levels points to other more plausible possibilities. Namely, the increase in CBS levels observed with age may be necessary for the production of $\mathrm{H}_{2} \mathrm{~S}$, which is a regulator of the synthesis of anti- and pro-oxidant enzymes and mediators of inflammation (Ingenbleek and Kimura, 2013; Kabil et al., 2014a,b). In fact, it is known that various synthetic drugs, which supply this gas, have been used for the treatment of deafness with variable results (Lamm and Arnold, 1998; Mahmood et al., 2014). CBS also contributes to cysteine synthesis, a semi-essential proteinogenic amino acid that is mostly obtained from the diet although a $50 \%$ of that synthesized through transuslfuration is used in GSH synthesis. On the other hand, the increase of BHMT after supplementation seems to derive from the need to reduce/regulate betaine levels. Hydrolysis of phosphatidylcholine may lead, on one side, to fatty acids for the production of anti-inflammatory mediators and on the other to choline. This choline excess might be oxidized to produce the osmolyte betaine (Zeisel et al., 1980; Porter et al., 1993), and hence the need to regulate its concentrations through Hcy remethylation. As a result, the methionine cycle would recover one of the methylation equivalents used by PEMT for phosphatidylcholine synthesis, while methionine and AdoMet levels would be sustained. This possibility is supported by data obtained in the liver of animals supplemented with PUFAs, where increased expression of Pemt and Gnmt is detected; two methyltransferases expressed in the cochlea according to microarray data (Gene Expression Omnibus GSE11821; Sanchez-Calderon et al., 2010). Nevertheless, the limited cochlear effect of PUFAs supplementation was attributed to the use of a formulation especially rich in eicosapentaenoic acid, which is known to be more effective for reducing inflammation (Bhattacharya et al., 2007).

Recently, Brown et al. (2014) investigated the putative protection against noise injury exerted by the administration of nicotinamide riboside, a precursor of $\mathrm{NAD}^{+}$, a cofactor that is involved in the regulation of sirtuins. Defects in the function of mitochondrial SIRT3 deacetylase lead to generation of reactive oxygen species (He et al., 2012) and decreased GSH levels (Someya et al., 2010). Both are factors associated to susceptibility to noise-induced HL (Someya et al., 2010) and to the reduction of cochlear $\mathrm{NAD}^{+}$levels (Ohlemiller et al., 1999). Administration of nicotinamide riboside injected twice daily for 5 days prior to noise exposure and for $48 \mathrm{~h}$ thereafter until sacrifice, prevented noise-induced HL and spiral ganglia neurite degeneration. These effects were mediated by the $\mathrm{NAD}^{+}$-dependent SIRT3, since deletion of this gene abrogated protection by nicotinamide riboside and expression of $\mathrm{NAD}^{+}$biosynthetic enzymes, whereas SIRT3-overexpressing mice became resistant to noise-induced HL (Brown et al., 2014). Interestingly, AHCY requires $\mathrm{NAD}^{+}$ for activity, thus the use of this compound may also contribute to improve cochlear Hcy metabolism, an aspect that was not addressed by the authors and that requires further attention.

\section{CONCLUSIONS}

Here we review recent evidence supporting the concept that the onset and progression of SNHL are closely linked to the availability of nutrients and their metabolism. Cumulative epidemiological, genetic and experimental models studies point to Hcy metabolism as a central node in the response to otic damage, although much more work is needed to fully understand cochlear Hcy metabolism, how it is regulated by nutrition, impacted by aging or noxious stimuli. However, available data fully support the potential of nutritional therapy for the protection of HL progression.

\section{AUTHOR CONTRIBUTIONS}

TP and IV-N, drafted the manuscript; NV, GV-M and MAP reviewed the manuscript; All authors gave final approval for publication. 


\section{FUNDING}

This work was supported by the European Commission FP7-PEOPLE-2013-IAPP TARGEAR and Spanish Ministerio de Economía y Competitividad (MINECO)/FEDER SAF201453979-R to IV-N. NV holds a CSIC predoctoral contract associated to FP7-AFHELO.

\section{REFERENCES}

Behera, J., Bala, J., Nuru, M., Tyagi, S. C., and Tyagi, N. (2016). Homocysteine as a pathological biomarker for bone disease. J. Cell. Physiol. doi: 10.1002/jcp. 25693 [Epub ahead of print].

Bhattacharya, A., Sun, D., Rahman, M., and Fernandes, G. (2007). Different ratios of eicosapentaenoic and docosahexaenoic omega-3 fatty acids in commercial fish oils differentially alter pro-inflammatory cytokines in peritoneal macrophages from C57BL/6 female mice. J. Nutr. Biochem. 18, 23-30. doi: 10.1016/j.jnutbio.2006.02.005

Boot, M. J., Gittenberger-de Groot, A. C., Poelmann, R. E., and Gourdie, R. G. (2006). Connexin43 levels are increased in mouse neural crest cells exposed to homocysteine. Birth Defects Res. A Clin. Mol. Teratol. 76, 133-137. doi: 10.1002/bdra.20220

Brosnan, J. T., Jacobs, R. L., Stead, L. M., and Brosnan, M. E. (2004). Methylation demand: a key determinant of homocysteine metabolism. Acta Biochim. Pol. 51, 405-413.

Brown, K. D., Maqsood, S., Huang, J. Y., Pan, Y., Harkcom, W., Li, W., et al. (2014). Activation of SIRT3 by the $\mathrm{NAD}^{+}$precursor nicotinamide riboside protects from noise-induced hearing loss. Cell Metab. 20, 1059-1068. doi: 10.1016/j. cmet.2014.11.003

Cadoni, G., Agostino, S., Scipione, S., and Galli, J. (2004). Low serum folate levels: a risk factor for sudden sensorineural hearing loss? Acta Otolaryngol. 124, 608-611. doi: 10.1080/00016480410016216

Carrillo-Carrasco, N., Chandler, R. J., and Venditti, C. P. (2012). Combined methylmalonic acidemia and homocystinuria, cblC type. I. Clinical presentations, diagnosis and management. J. Inherit. Metab. Dis. 35, 91-102. doi: 10.1007/s10545-011-9365-x

Church, M. W., Jen, K.-L. C., Anumba, J. I., Jackson, D. A., Adams, B. R., and Hotra, J. W. (2010). Excess omega-3 fatty acid consumption by mothers during pregnancy and lactation caused shorter life span and abnormal ABRs in old adult offspring. Neurotoxicol. Teratol. 32, 171-181. doi: 10.1016/j.ntt.2009. 09.006

Church, M. W., Jen, K.-L. C., Jackson, D. A., Adams, B. R., and Hotra, J. W. (2009). Abnormal neurological responses in young adult offspring caused by excess omega-3 fatty acid (fish oil) consumption by the mother during pregnancy and lactation. Neurotoxicol. Teratol. 31, 26-33. doi: 10.1016/j.ntt.2008.09.001

Church, M. W., Jen, K. L., Stafferton, T., Hotra, J. W., and Adams, B. R. (2007). Reduced auditory acuity in rat pups from excess and deficient omega-3 fatty acid consumption by the mother. Neurotoxicol. Teratol. 29, 203-210. doi: 10.1016/j.ntt.2006.10.009

Cohen-Salmon, M., Regnault, B., Cayet, N., Caille, D., Demuth, K., Hardelin, J. P., et al. (2007). Connexin30 deficiency causes instrastrial fluid-blood barrier disruption within the cochlear stria vascularis. Proc. Natl. Acad. Sci. U S A 104, 6229-6234. doi: 10.1073/pnas.0605108104

Curhan, S. G., Eavey, R. D., Wang, M., Rimm, E. B., and Curhan, G. C. (2014). Fish and fatty acid consumption and the risk of hearing loss in women. Am. J. Clin. Nutr. 100, 1371-1377. doi: 10.3945/ajcn.114.091819

Dror, A. A., and Avraham, K. B. (2009). Hearing loss: mechanisms revealed by genetics and cell biology. Annu. Rev. Genet. 43, 411-437. doi: 10.1146/annurevgenet-102108-134135

Dullemeijer, C., Verhoef, P., Brouwer, I. A., Kok, F. J., Brummer, R.-J. M., and Durga, J. (2010). Plasma very long-chain n-3 polyunsaturated fatty acids and age-related hearing loss in older adults. J. Nutr. Health Aging 14, 347-351. doi: 10.1007/s12603-010-0078-x

Durga, J., Anteunis, L. J., Schouten, E. G., Bots, M. L., Kok, F. J., and Verhoef, P. (2006). Association of folate with hearing is dependent

\section{ACKNOWLEDGMENTS}

The authors thank Javier Pérez (IIBM, CSIC-UAM) for the technical support provided. We are grateful to our present and former colleagues of the Neurobiology of Hearing group for sharing information and useful discussion.

on the 5,10-methylenetetrahdyrofolate reductase $677 \mathrm{C} \rightarrow \mathrm{T}$ mutation Neurobiol. Aging 27, 482-489. doi: 10.1016/j.neurobiolaging.2005. 03.002

Durga, J., Verhoef, P., Anteunis, L. J., Schouten, E., and Kok, F. J. (2007). Effects of folic acid supplementation on hearing in older adults: a randomized, controlled trial. Ann. Intern. Med. 146, 1-9. doi: 10.7326/0003-4819-146-1-20070102000003

Fetoni, A. R., Eramo, S. L., Paciello, F., Rolesi, R., Podda, M. V., Troiani, D., et al. (2014). Curcuma longa (curcumin) decreases in vivo cisplatininduced ototoxicity through heme oxygenase-1 induction. Otol. Neurotol. 35, e169-e177. doi: 10.1097/MAO.0000000000000302

Fetoni, A. R., Mancuso, C., Eramo, S. L., Ralli, M., Piacentini, R., Barone, E., et al. (2010). In vivo protective effect of ferulic acid against noise-induced hearing loss in the guinea-pig. Neuroscience 169, 1575-1588. doi: 10.1016/j. neuroscience.2010.06.022

Fetoni, A. R., Paciello, F., Rolesi, R., Eramo, S. L., Mancuso, C., Troiani, D., et al (2015). Rosmarinic acid up-regulates the noise-activated Nrf2/HO-1 pathway and protects against noise-induced injury in rat cochlea. Free Radic. Biol. Med. 85, 269-281. doi: 10.1016/j.freeradbiomed.2015.04.021

Fusconi, M., Chistolini, A., de Virgilio, A., Greco, A., Massaro, F., Turchetta, R., et al. (2012). Sudden sensorineural hearing loss: a vascular cause? Analysis of prothrombotic risk factors in head and neck. Int. J. Audiol. 51, 800-805. doi: 10.3109/14992027.2012.705904

Givvimani, S., Munjal, C., Narayanan, N., Aqil, F., Tyagi, G., Metreveli, N., et al. (2012). Hyperhomocysteinemia decreases intestinal motility leading to constipation. Am. J. Physiol. Gastrointest. Liver Physiol. 303, G281-G290. doi: 10.1152/ajpgi.00423.2011

Global Burden of Disease Study 2013 Collaborators. (2015). Global, regional, and national incidence, prevalence, and years lived with disability for 301 acute and chronic diseases and injuries in 188 countries, 1990-2013: a systematic analysis for the Global burden of disease study 2013. Lancet 386, 743-800. doi: 10.1016/S0140-6736(15)60692-4

Gok, U., Halifeoglu, I., Canatan, H., Yildiz, M., Gursu, M. F., and Gur, B. (2004). Comparative analysis of serum homocysteine, folic acid and vitamin B12 levels in patients with noise-induced hearing loss. Auris Nasus Larynx 31, 19-22. doi: 10.1016/j.anl.2003.09.001

Gopinath, B., Flood, V. M., Rochtchina, E., McMahon, C. M., and Mitchell, P. (2010). Consumption of omega-3 fatty acids and fish and risk of age-related hearing loss. Am. J. Clin. Nutr. 92, 416-421. doi: 10.3945/ajen.2010.29370

Gordon, A. M., Capute, A. J., and Konigsmark, B. W. (1976). Progressive quadriparesis, mental retardation, retinitis pigmentosa, and hearing loss: report of two sibs. Johns Hopkins Med. J. 138, 142-145.

Gratton, M. A., and Schulte, B. A. (1995). Alterations in microvasculature are associated with atrophy of the stria vascularis in quiet-aged gerbils. Hear. Res. 82, 44-52. doi: 10.1016/0378-5955(94)00161-i

Harding, C. O., Pillers, D. A., Steiner, R. D., Bottiglieri, T., Rosenblatt, D. S. Debley, J., et al. (2003). Potential for misdiagnosis due to lack of metabolic derangement in combined methylmalonic aciduria/hyperhomocysteinemia (cblC) in the neonate. J. Perinatol. 23, 384-386. doi: 10.1038/sj.jp.72 10955

Hartikka, H., Kuurila, K., Körkkö, J., Kaitila, I., Grénman, R., Pynnönen, S., et al. (2004). Lack of correlation between the type of COL1A1 or COL1A2 mutation and hearing loss in osteogenesis imperfecta patients. Hum. Mutat. 24, 147-154 doi: 10.1002/humu.20108

He, W., Newman, J. C., Wang, M. Z., Ho, L., and Verdin, E. (2012). Mitochondrial sirtuins: regulators of protein acylation and metabolism. Trends Endocrinol. Metab. 23, 467-476. doi: 10.1016/j.tem.2012.07.004 
Houston, D. K., Johnson, M. A., Nozza, R. J., Gunter, E. W., Shea, K. J., Cutler, G. M., et al. (1999). Age-related hearing loss, vitamin B-12, and folate in elderly women. Am. J. Clin. Nutr. 69, 564-571.

Huang, T., Hu, X., Khan, N., Yang, J., and Li, D. (2013). Effect of polyunsaturated fatty acids on homocysteine metabolism through regulating the gene expressions involved in methionine metabolism. ScientificWorldJournal 2013:931626. doi: 10.1155/2013/931626

Huang, T., Zheng, J., Chen, Y., Yang, B., Wahlqvist, M. L., and Li, D. (2011). High consumption of $\Omega-3$ polyunsaturated fatty acids decrease plasma homocysteine: a meta-analysis of randomized, placebo-controlled trials. Nutrition 27, 863-867. doi: 10.1016/j.nut.2010.12.011

Iacobazzi, V., Infantino, V., Castegna, A., and Andria, G. (2014). Hyperhomocysteinemia: related genetic diseases and congenital defects, abnormal DNA methylation and newborn screening issues. Mol. Genet. Metab. 113, 27-33. doi: 10.1016/j.ymgme.2014.07.016

Ingenbleek, Y., and Kimura, H. (2013). Nutritional essentiality of sulfur in health and disease. Nutr. Rev. 71, 413-432. doi: 10.1111/nure.12050

Jacques, P. F., Selhub, J., Bostom, A. G., Wilson, P. W., and Rosenberg, I. H. (1999). The effect of folic acid fortification on plasma folate and total homocysteine concentrations. $N$ Engl. J. Med. 340, 1449-1454. doi: 10.1056/nejm199905133401901

Johnsson, L. G., and Hawkins, J. E. Jr. (1972). Vascular changes in the human inner ear associated with aging. Ann. Otol. Rhinol. Laryngol. 81, 364-376. doi: $10.1177 / 000348947208100307$

Karli, R., Gül, A., and Uğur, B. (2013). Effect of vitamin B12 deficiency on otoacoustic emissions. Acta Otorhinolaryngol. Ital. 33, 243-247.

Kabil, O., Motl, N., and Banerjee, R. (2014a). $\mathrm{H}_{2} \mathrm{~S}$ and its role in redox signaling. Biochim. Biophys. Acta 1844, 1355-1366. doi: 10.1016/j.bbapap.2014.01.002

Kabil, O., Vitvitsky, V., and Banerjee, R. (2014b). Sulfur as a signaling nutrient through hydrogen sulfide. Annu. Rev. Nutr. 34, 171-205. doi: 10.1146/annurevnutr-071813-105654

Kulkarni, A., Dangat, K., Kale, A., Sable, P., Chavan-Gautam, P., and Joshi, S. (2011). Effects of altered maternal folic acid, vitamin $B 12$ and docosahexaenoic acid on placental global DNA methylation patterns in Wistar rats. PLoS One 6:e17706. doi: 10.1371/journal.pone.0017706

Kundu, S., Munjal, C., Tyagi, N., Sen, U., Tyagi, A. C., and Tyagi, S. C. (2012). Folic acid improves inner ear vascularization in hyperhomocysteinemic mice. Hear. Res. 284, 42-51. doi: 10.1016/j.heares.2011.12.006

Kundu, S., Tyagi, N., Sen, U., and Tyagi, S. C. (2009). Matrix imbalance by inducing expression of metalloproteinase and oxidative stress in cochlea of hyperhomocysteinemic mice. Mol. Cell. Biochem. 332, 215-224. doi: 10.1007/s11010-009-0194-2

Lamm, K., and Arnold, W. (1998). The effect of prednisolone and non-steroidal anti-inflammatory agents on the normal and noise-damaged guinea pig inner ear. Hear Res. 115, 149-161. doi: 10.1016/s0378-5955(97)00186-x

Lasisi, A. O., Fehintola, F. A., and Yusuf, O. B. (2010). Age-related hearing loss, vitamin B12, and folate in the elderly. Otolaryngol. Head Neck Surg. 143, 826-830. doi: 10.1016/j.otohns.2010.08.031

Lehotsky, J., Petras, M., Kovalska, M., Tothova, B., Drgova, A., and Kaplan, P. (2015). Mechanisms involved in the ischemic tolerance in brain: effect of the homocysteine. Cell. Mol. Neurobiol. 35, 7-15. doi: 10.1007/s10571-014$0112-3$

Lei, W., Long, Y., Li, S., Liu, Z., Zhu, F., Hou, F. F., et al. (2015). Homocysteine induces collagen I expression by downregulating histone methyltransferase G9a. PLoS One 10:e0130421. doi: 10.1371/journal.pone.0130421

Li, H., Brodsky, S., Kumari, S., Valiunas, V., Brink, P., Kaide, J., et al. (2002). Paradoxical overexpression and translocation of connexin 43 in homocysteinetreated endothelial cells. Am. J. Physiol. Heart Circ. Physiol. 282, H2124-H2133. doi: 10.1152/ajpheart.01028.2001

Li, X., Mao, X. B., Hei, R. Y., Zhang, Z. B., Wen, L. T., Zhang, P. Z., et al. (2011). Protective role of hydrogen sulfide against noise-induced cochlear damage: a chronic intracochlear infusion model. PLoS One 6:e26728. doi: 10.1371/journal. pone. 0026728

Li-Korotky, H. S. (2012). Age-related hearing loss: quality of care for quality of life. Gerontologist 52, 265-271. doi: 10.1093/geront/gnr159

Lipton, S. A., Kim, W.-K., Choi, Y.-B., Kumar, S., D’Emilia, D. N., Rayudu, P. V., et al. (1997). Neurotoxicity associated with dual actions of homocysteine at the N-methyl-D-aspartate receptor. Proc. Natl. Acad. Sci. U S A 94, 5923-5928. doi: $10.1073 /$ pnas. 94.11 .5923
Magariños, M., Contreras, J., Aburto, M. R., and Varela-Nieto, I. (2012). Early development of the vertebrate inner ear. Anat. Rec. (Hoboken) 295, 1775-1790. doi: 10.1002/ar.22575

Magariños, M., Contreras, J., and Varela-Nieto, I. (2014). "Early development of the vertebrate inner ear," in Development of the Auditory and Vestibular Systems, eds R. Romand and I. Varela-Nieto (Amsterdam-Boston: Elservier), $1-30$.

Mahmood, G., Mei, Z., Hojjat, H., Pace, E., Kallakuri, S., and Zhang, J. S. (2014). Therapeutic effect of sildenafil on blast-induced tinnitus and auditory impairment. Neuroscience 269, 367-382. doi: 10.1016/j.neuroscience.2014. 03.020

Makishima, K. (1978). Arteriolar sclerosis as a cause of presbycusis. Otolaryngology 86, ORL322-ORL326. doi: 10.1177/019459987808600225

Mancuso, C. (2015). Key factors which concur to the correct therapeutic evaluation of herbal products in free radical-induced diseases. Front. Pharmacol. 6:86. doi: 10.3389/fphar.2015.00086

Martínez-Vega, R., Garrido, F., Partearroyo, T., Cediel, R., Zeisel, S. H., MartínezÁlvarez, C., et al. (2015a). Folic acid deficiency induces premature hearing loss through mechanisms involving cochlear oxidative stress and impairment of homocysteine metabolism. FASEB J. 29, 418-432. doi: 10.1096/fj.14-259283

Martínez-Vega, R., Partearroyo, T., Vallecillo, N., Varela-Moreiras, G., Pajares, M. A., and Varela-Nieto, I. (2015b). Long-term omega-3 fatty acid supplementation prevents expression changes in cochlear homocysteine metabolism and ameliorates progressive hearing loss in C57BL/6J mice. J. Nutr. Biochem. 26, 1424-1433. doi: 10.1016/j.jnutbio.2015.07.011

Martínez-Vega, R., Murillo-Cuesta, S., Partearroyo, T., Varela-Moreiras, G., Varela-Nieto, I., and Pajares, M. A. (2016). Long-term dietary folate deficiency accelerates progressive hearing loss on CBA/Ca mice. Front. Aging Neurosci. 8:209. doi: 10.3389/fnagi.2016.00209

McCully, K. S. (1969). Vascular pathology of homocysteinemia: implications for the pathogenesis of arteriosclerosis. Am. J. Pathol. 56, 111-128.

Noga, A. A., Stead, L. M., Zhao, Y., Brosnan, M. E., Brosnan, J. T., and Vance, D. E. (2003). Plasma homocysteine is regulated by phospholipid methylation. J. Biol. Chem. 278, 5952-5955. doi: 10.1074/jbc.M212194200

Obeid, R. (2013). The metabolic burden of methyl donor deficiency with focus on the betaine homocysteine methyltransferase pathway. Nutrients 5, 3481-3495. doi: 10.3390/nu5093481

Ohlemiller, K. K., Wright, J. S., and Dugan, L. L. (1999). Early elevation of cochlear reactive oxygen species following noise exposure. Audiol. Neurootol. 4, 229-236. doi: 10.1159/000013846

Pajares, M. A., and Pérez-Sala, D. (2006). Betaine homocysteine S-methyltransferase: just a regulator of homocysteine metabolism? Cell. Mol. Life Sci. 63, 2792-2803. doi: 10.1007/s00018-006-6249-6

Perna, A. F., and Ingrosso, D. (2016). Atherosclerosis determinants in renal disease: how much is homocysteine involved? Nephrol. Dial. Transplant 31, 860-863. doi: 10.1093/ndt/gfv409

Piolot, A., Blache, D., Boulet, L., Fortin, L. J., Dubreuil, D., Marcoux, C., et al. (2003). Effect of fish oil on LDL oxidation and plasma homocysteine concentrations in health. J. Lab. Clin. Med. 141, 41-49. doi: 10.1067/mlc. 2003.3

Porter, R. K., Scott, J. M., and Brand, M. D. (1993). Characterization of betaine efflux from rat liver mitochondria. Biochim. Biophys. Acta 1141, 269-274. doi: 10.1016/0005-2728(93)90052-h

Prazma, J., Carrasco, V. N., Butler, B., Waters, G., Anderson, T., and Pillsbury, H. C. (1990). Cochlear microcirculation in young and old gerbils. Arch. Otolaryngol. Head Neck Surg. 116, 932-936. doi: 10.1001/archotol.1990. 01870080054015

Puel, J. L., Ladrech, S., Chabert, R., Pujol, R., and Eybalin, M. (1991). Electrophysiological evidence for the presence of NMDA receptors in the guinea pig cochlea. Hear Res. 51, 255-264. doi: 10.1016/0378-5955(91) 90042-8

Refsum, H., Helland, S., and Ueland, P. M. (1989). Fasting plasma homocysteine as a sensitive parameter of antifolate effect: a study of psoriasis patients receiving low-dose methotrexate treatment. Clin. Pharmacol. Ther. 46, 510-520. doi: 10.1038/clpt.1989.179

Rosen, S., and Olin, P. (1965). Hearing loss and coronary heart disease. Bull. N Y Acad. Med. 41, 1052-1068

Rosen, S., Olin, P., and Rosen, H. V. (1970). Dietary prevention of hearing loss. Acta Otolaryngol. 70, 242-247. doi: 10.3109/00016487009181884 
Roth, T. N., Hanebuth, D., and Probst, R. (2011). Prevalence of age-related hearing loss in Europe: a review. Eur. Arch. Otorhinolaryngol. 268, 1101-1107. doi: 10.1007/s00405-011-1597-8

Rydzanicz, M., Wróbel, M., Pollak, A., Gawecki, W., Brauze, D., KostrzewskaPoczekaj, M., et al. (2010). Mutation analysis of mitochondrial 12S rRNA gene in Polish patients with non-syndromic and aminoglycoside-induced hearing loss. Biochem. Biophys. Res. Commun. 395, 116-121. doi: 10.1016/j.bbrc.2010. 03.149

Sanchez-Calderon, H., Rodriguez-de la Rosa, L., Milo, M., Pichel, J. G., Holley, M., and Varela-Nieto, I. (2010). RNA microarray analysis in prenatal mouse cochlea reveals novel IGF-I target genes: implication of MEF2 and FOXM1 transcription factors. PLoS One 5:e8699. doi: 10.1371/journal.pone. 0008699

Schalinske, K. L., and Smazal, A. L. (2012). Homocysteine imbalance: a pathological metabolic marker. Adv. Nutr. 3, 755-762. doi: 10.3945/an.112. 002758

Schuknecht, H. F., Watanuki, K., Takahashi, T., Belal, A. A. Jr., Kimura, R. S., Jones, D. D., et al. (1974). Atrophy of the stria vascularis, a common cause for hearing loss. Laryngoscope 84, 1777-1821. doi: 10.1288/00005537-19741000000012

Sharma, G. S., Kumar, T., Dar, T. A., and Singh, L. R. (2015). Protein Nhomocysteinylation: from cellular toxicity to neurodegeneration. Biochim. Biophys. Acta 1850, 2239-2245. doi: 10.1016/j.bbagen.2015.08.013

Someya, S., Yu, W., Hallows, W. C., Xu, J., Vann, J. M., Leeuwenburgh, C., et al. (2010). Sirt3 mediates reduction of oxidative damage and prevention of age-related hearing loss under caloric restriction. Cell 143, 802-812. doi: 10.1016/j.cell.2010.10.002

Stefanutti, C., Morozzi, C., Di Giacomo, S., and Italian Multicenter Study on Low-Density Lipoprotein Apheresis Working Group. (2013). Italian multicenter study on low-density lipoprotein apheresis Working Group 2009 survey. Ther. Apher. Dial. 17, 169-178. doi: 10.1111/j.1744-9987.2012. 01142.x

Tabuchi, K., Nishimura, B., Nakamagoe, M., Hayashi, K., Nakayama, M., and Hara, A. (2011). Ototoxicity: mechanisms of cochlear impairment and its prevention. Curr. Med. Chem. 18, 4866-4871. doi: $10.2174 / 092986711797535254$
Tsai, A. C.-H., Morel, C. F., Scharer, G., Yang, M., Lerner-Ellis, J. P., Rosenblatt, D. S., et al. (2007). Late-onset combined homocystinuria and methylmalonic aciduria $(\mathrm{cblC})$ and neuropsychiatric disturbance. Am. J. Med. Genet. A 143A, 2430-2434. doi: 10.1002/ajmg.a.31932

Uchida, Y., Sugiura, S., Ando, F., Nakashima, T., and Shimokata, H. (2011). Hearing impairment risk and interaction of folate metabolism related gene polymorphisms in an aging study. BMC Med. Genet. 12:35. doi: 10.1186/14712350-12-35

Ueland, P. M., and Refsum, H. (1989). Plasma homocysteine, a risk factor for vascular disease: plasma levels in health, disease and drug therapy. J. Lab. Clin. Med. 114, 473-501.

World Health Organization. (2015). Deafness and hearing loss. Available online at: http://www.who.int/mediacentre/factsheets/fs300/en/

Yang, B., Fan, S., Zhi, X., Wang, Y., Wang, Y., Zheng, Q., et al. (2014). Prevalence of hyperhomocysteinemia in China: a systematic review and meta-analysis. Nutrients 7, 74-90. doi: 10.3390/nu7010074

Zeisel, S. H., Story, D. L., Wurtman, R. J., and Brunengraber, H. (1980). Uptake of free choline by isolated perfused rat liver. Proc. Natl. Acad. Sci. U S A 77, 4417-4419. doi: 10.1073/pnas.77.8.4417

Zhao, H., Li, R., Wang, Q., Yan, Q., Deng, J. H., Han, D., et al. (2004). Maternally inherited aminoglycoside-induced and nonsyndromic deafness is associated with the novel C1494T mutation in the mitochondrial $12 \mathrm{~S}$ rRNA gene in a large Chinese family. Am. J. Hum. Genet. 74, 139-152. doi: 10.1086/ 381133

Conflict of Interest Statement: The authors declare that the research was conducted in the absence of any commercial or financial relationships that could be construed as a potential conflict of interest.

Copyright (C) 2017 Partearroyo, Vallecillo, Pajares, Varela-Moreiras and Varela-Nieto. This is an open-access article distributed under the terms of the Creative Commons Attribution License (CC BY). The use, distribution or reproduction in other forums is permitted, provided the original author(s) or licensor are credited and that the original publication in this journal is cited, in accordance with accepted academic practice. No use, distribution or reproduction is permitted which does not comply with these terms. 